\title{
Schwangerschaftsplanung bei bestehender Hypertonie
}

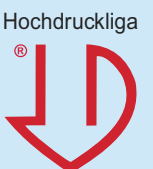

In Zusammenarbeit mit der Deutschen Hochdruckliga e.V. DHL ${ }^{\oplus}$ - Deutsche Gesellschaft für Hypertonie und Prävention

Vorsitzender: Prof. Dr. med. U. Kintscher, Berlin

\section{Anamnese}

Die 35jährige Patientin stellte sich nachts mit einer hypertensiven Entgleisung (Blutdruck 200/110 mmHg) in der Notaufnahme vor. Am Vortag sei ein Migräneanfall mit Erbrechen aufgetreten. Sie habe dann mit dem Blutdruckmessgerät der Mutter einen Blutdruck von 180/100 mmHg gemessen. Bluthochdruck sei bisher bei ihr nicht bekannt, beide Eltern nähmen aber Medikamente dagegen. Eine erste Schwangerschaft vor zehn Jahren sei unauffällig verlaufen, sie habe jedoch währenddessen sehr viel zugenommen. Innerhalb des letzten Jahres habe sie aber $10 \mathrm{~kg}$ abgenommen. Die Pille habe sie vor zwei Monaten abgesetzt, da Sie wieder schwanger werden wolle.

\section{Weitere Klinische Untersuchung}

- Größe 164 cm, Gewicht 94 kg, BMI 34,9 $\mathrm{kg} / \mathrm{m}^{2}$

- Klinischer Status ansonsten unauffällig

\section{Abdomensonographie}

Niere links 11,00 x 5,8 cm, Parenchymdicke $1,29 \mathrm{~cm}$, Niere rechts $10,8 \times 4,5 \mathrm{~cm}$, Parenchymdicke $1,54 \mathrm{~cm}$, beidseits glatte Oberfläche, kein Aufstau.

\section{Laborbefunde}

- Kreatinin 0,83 mg/dl (0,5-0,9)

- Harnstoff-N 25 mg/dl (10-50)

- Kalium 4,3 mmol/1 (3,5-5,1)

- Glukose 87 mg/dl (55-110)

- Cholesterin 186 mg/dl (150-200)

- Triglyzeride $117 \mathrm{mg} / \mathrm{dl}(70-170)$

- HDL 42 mg/dl (> 40, optimal > 60)

- LDL $133 \mathrm{mg} / \mathrm{dl}$ (<100 optimal)

- CRP 0,5 mg/dl $(<0,5)$

- Hb 15,0 g/dl (12,0-116,0)

- Leukozyten 9,3/nl (4,0-10,0)

\section{Urinstatus}

- $\mathrm{pH} 6,5$

- Leukozyten und Erythrozyten negativ

- Albumin 250 mg/g Kreatinin (<28)

- Kreatinin-Clearance (MDRD) $83 \mathrm{ml} / \mathrm{min}$

\section{Weitere Abklärung}

In einer ambulanten Langzeitblutdruckmessung bestätigte sich eine arterielle $\mathrm{Hy}$ pertonie WHO-Stadium 2, mit durchschnittlichen Blutdruckwerten von 173/122 mmHg und fehlender Nachtabsenkung. Weitere Untersuchungen zum Ausschluss einer sekundären Genese des Bluthochdrucks blieben ohne richtungweisenden Befund. Das EKG zeigte eine linksventrikuläre Hypertrophie, die sich in einer Echokardiografie bestätigte.

\section{Therapie}

In der Nacht wurde der Blutdruck mit 5 mg Amlodipin auf 170/100 mmHg gesenkt. Die Patientin erhielt eine Diätberatung mit dem Ziel, eine Kochsalz-arme Kost einzuhalten und das Gewicht weiter zu reduzieren, auch sportliche Betätigung wurde empfohlen. In Anbetracht des auch von ihr selbst wahrgenommenen Risikos durch die Kombination von Adipositas und Hypertonie mit bereits feststellbaren Endorganschäden, entschied sich die $\mathrm{Pa}$ tientin, von einer erneuten Schwangerschaft Abstand zu nehmen, bis der Blutdruck gut behandelt und eine Gewichtsreduktion erreicht sei. Die medikamentöse Therapie wurde mit einem KalziumAntagonisten eingeleitet.

\section{Kommentar}

Die Prävalenz chronischer Hypertonie in der Schwangerschaft - definiert als Blutdruckwerte von $\geq 140 \mathrm{mmHg}$ systolisch und $\geq 90 \mathrm{mmHg}$ diastolisch gemessen vor der 20. Schwangerschaftswoche und sechs Wochen über die Geburt hinausgehend nimmt vor allem in industrialisierten Ländern zu. Hypertonikerinnen, die schwanger werden, haben ein erhöhtes Risiko für Schwangerschaftskomplikationen, deren Häufigkeit und Schwere mit der Schwere der Hypertonie steigt. Zu den potentiellen Komplikationen gehören die Präeklampsie / Eklampsie, Frühgeburtlichkeit, niedriges
Geburtsgewicht der Kinder, Notwendigkeit einer Sectio und der Plazentaabriss. Zur Vorsorge für Hypertonikerinnen, die schwanger werden möchten, gehört die Evaluation möglicher Endorganschäden, insbesondere der Nierenfunktion, da eine bereits vor Eintreten einer Schwangerschaft bestehende Proteinurie das Präeklampsierisiko weiter erhöht, die Diagnose aber gleichzeitig erschwert. Zur medikamentösen Blutdruckeinstellung ist Alphamethyldopa weiterhin Mittel der ersten Wahl, Betablocker und inzwischen auch Kalzium-Antagonisten werden ebenfalls zur oralen Bluthochdrucktherapie in der Schwangerschaft eingesetzt. Dihydralazin und Urapidil können bei schwerer Hypertonie verwendet werden, gehören jedoch nicht zur oralen Therapie der ersten Wahl. ACE-Hemmer und $\mathrm{AT}_{1}$-Blocker sind wegen ihrer embryo- und fetotoxischen Effekte kontraindiziert und sollten vor einer geplanten Schwangerschaft ersetzt werden. Bei adipösen Patientinnen gehört eine Gewichtsreduktion vor der Schwangerschaft zu den empfohlenen Maßnahmen, nicht jedoch während der Schwangerschaft, da sich dies ungünstig auf das Wachstum des Kindes auswirken kann. Auch eine zu strenge Blutdruckeinstellung während der Schwangerschaft kann zu Untergewicht und Wachstumsretardierung des Kindes führen. Die Leitlinien der Deutschen Hochdruckliga empfehlen heute für schwangere Hypertonikerinnen Zielblutdruckwerte zwischen 140-160 und 90-100 $\mathrm{mmHg}$. Eine milde Hypertonie im WHOStadium 1 wird danach nicht medikamentös behandelt.

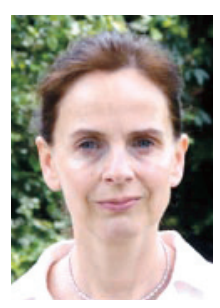

Korrespondenzadresse: PD Dr. Anna Mitchell Medizinische Klinik I Marienhospital Herne Ruhr-Universität Bochum Hölkeskampring 40 44625 Herne E-Mail: anna.mitchell@ marienhospital-herne.de 